\title{
The effects of capillary dysfunction on oxygen and glucose extraction in diabetic neuropathy
}

\author{
Leif Østergaard • Nanna B. Finnerup • Astrid J. Terkelsen • Rasmus A. Olesen • \\ Kim R. Drasbek • Lone Knudsen • Sune N. Jespersen • Jan Frystyk • \\ Morten Charles • Reimar W. Thomsen • Jens S. Christiansen • \\ Henning Beck-Nielsen • Troels S. Jensen • Henning Andersen
}

Received: 14 September 2014 / Accepted: 6 November 2014 / Published online: 16 December 2014

(C) The Author(s) 2014. This article is published with open access at Springerlink.com

\begin{abstract}
Diabetic neuropathy is associated with disturbances in endoneurial metabolism and microvascular morphology, but the roles of these factors in the aetiopathogenesis of diabetic neuropathy remain unclear. Changes in endoneurial capillary morphology and vascular reactivity apparently predate the development of diabetic neuropathy in humans, and in manifest neuropathy, reductions in nerve conduction velocity correlate with the level of endoneurial hypoxia. The idea that microvascular changes cause diabetic neuropathy is contradicted, however, by reports of elevated endoneurial blood flow in early experimental diabetes, and of unaffected blood flow when early histological signs of neuropathy first develop in humans. We recently showed that disturbances in capillary flow patterns, so-called capillary dysfunction, can reduce the amount of oxygen and glucose that can be extracted
\end{abstract}

\footnotetext{
L. Østergaard $(\bowtie) \cdot$ R. A. Olesen $\cdot$ K. R. Drasbek $\cdot$ S. N. Jespersen Center of Functionally Integrative Neuroscience and MINDLab, Institute of Clinical Medicine, Aarhus University Hospital, Building 10G, Nørrebrogade 44, DK-8000 Aarhus C, Denmark

e-mail: leif@cfin.au.dk

\section{L. Østergaard}

Department of Neuroradiology, Aarhus University Hospital, Aarhus, Denmark

N. B. Finnerup · A. J. Terkelsen • L. Knudsen • T. S. Jensen Danish Pain Research Center, Institute of Clinical Medicine, Aarhus University, Aarhus, Denmark
}

\section{A. J. Terkelsen • T. S. Jensen $\cdot$ H. Andersen}

Department of Neurology, Aarhus University Hospital, Aarhus, Denmark

\section{Knudsen}

Spinal Cord Injury Centre, Department of Neurology, Viborg

Regional Hospital, Viborg, Denmark by the tissue for a given blood flow. In fact, tissue blood flow must be adjusted to ensure sufficient oxygen extraction as capillary dysfunction becomes more severe, thereby changing the normal relationship between tissue oxygenation and blood flow. This review examines the evidence of capillary dysfunction in diabetic neuropathy, and whether the observed relation between endoneurial blood flow and nerve function is consistent with increasingly disturbed capillary flow patterns. The analysis suggests testable relations between capillary dysfunction, tissue hypoxia, aldose reductase activity, oxidative stress, tissue inflammation and glucose clearance from blood. We discuss the implications of these predictions in relation to the prevention and management of diabetic complications in type 1 and type 2 diabetes, and suggest ways of testing these hypotheses in experimental and clinical settings.

\section{S. N. Jespersen}

Department of Physics and Astronomy, Aarhus University, Aarhus, Denmark

J. Frystyk $\cdot$ J. S. Christiansen

Department of Endocrinology and Diabetes, Aarhus University Hospital, Aarhus, Denmark

M. Charles

Department of Clinical Epidemiology, Aarhus University Hospital, Aarhus, Denmark

R. W. Thomsen

Department of Public Health, Aarhus University, Aarhus, Denmark

H. Beck-Nielsen

Department of Endocrinology, Odense University Hospital, Odense, Denmark

H. Beck-Nielsen

Institute of Clinical Research, University of Southern Denmark, Odense, Denmark 
Keywords Capillary dysfunction - Diabetic complications · Diabetic neuropathy - Glucose intolerance - Glucose transport - Microvascular disease

$\begin{array}{ll}\text { Abbreviations } \\ \text { BDNF } & \text { Brain-derived neurotrophic factor } \\ \text { CTH } & \text { Capillary transit time heterogeneity } \\ \text { DNP } & \text { Distal symmetric sensorimotor polyneuropathy } \\ \text { HIF-1 } & \text { Hypoxia inducible factor 1 } \\ \text { MTT } & \text { Mean erythrocyte transit time } \\ \text { NF-KB } & \text { Nuclear factor-kB } \\ \text { OEF } & \text { Oxygen extraction fraction } \\ \text { ROS } & \text { Reactive oxygen species } \\ \text { STZ } & \text { Streptozotocin } \\ \text { TBF } & \text { Tissue blood flow } \\ \text { tPA } & \text { Tissue plasminogen activator }\end{array}$

\section{Introduction}

Diabetic neuropathy affects up to $50 \%$ of patients with diabetes [1]. Distal symmetric sensorimotor polyneuropathy (DNP) is by far the most common form, carrying a high risk of foot ulcers and limb amputation [1]. In addition, one third of patients with neuropathy develop pain, with severe consequences for their quality of life [1,2]. Despite being the most common complication of diabetes, the pathophysiological mechanisms underlying diabetic neuropathy are largely unknown. The scientific community has generally been divided into two schools of thought, one of which favours a metabolic mechanism, and one proposing a vascular origin of diabetic neuropathy [3]. The latter hypothesis is founded in observations that diabetic neuropathy is associated with microvascular changes in the affected nerve trunks. Nerve biopsies reveal capillary basement membrane thickening, loss of capillary pericyte coverage, and endothelial hyperplasia [4] in endoneurial microvessels (Fig. 1). In fact, changes in endoneurial capillary density and luminal area appear to precede the development of impaired glucose tolerance and diabetes [5]. Nutritive perfusion is reduced in nerve trunks affected by diabetic neuropathy, and their conduction velocities are typically reduced in proportion to the reduction in their oxygen tension $[6,7]$. Indeed, changes in vascular reactivity can be recorded prior to the onset of hyperglycaemia in individuals at risk of type 2 diabetes [8]. This 'vascular' hypothesis is contradicted, however, by observations that endoneurial blood flow is elevated early after the induction of experimental diabetes in rats $[9,10]$, and observations that sural nerve blood flow in patients with mild diabetes remained constant over a 1-year time period during which nerve fibre density decreased [11]. Meanwhile, several metabolic pathways have been shown to cause nerve damage [12], and it
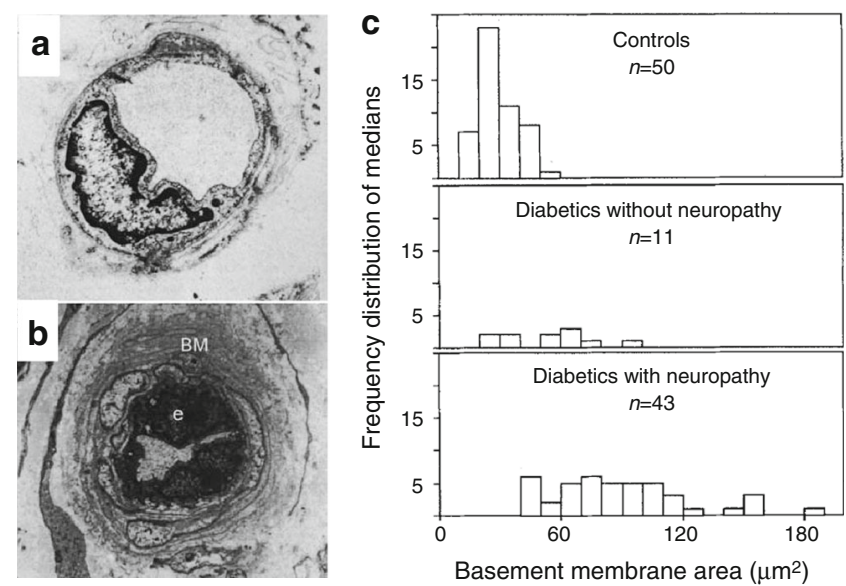

Fig. 1 (a, b) Endoneurial capillaries from the sural nerve of a diabetic patient without neuropathy (a) and a patient with neuropathy (b). Note basement membrane (BM) thickening and endothelial cell [e] proliferation in (b). Reproduced from [6] with permission from the publisher. (c) Histograms of the median basement membrane area (in $\mu \mathrm{m}^{2}$ ) of endoneurial microvessels in 54 diabetic patients ( 25 with type 1 diabetes, 29 with type 2 diabetes) and 50 controls. The areas were based on transverse electron micrographs of 433 microvessels from diabetic patients and 366 from controls. Note that basement membrane thickening is particularly prevalent in diabetes with neuropathy. Reproduced from Giannini and Dyck [4] with permission from the publisher

therefore appears that both vascular and metabolic mechanisms may be involved in the pathogenesis of diabetic neuropathy $[1,6]$.

We recently showed that if capillary flow patterns become disturbed, then the transit times of portions of the blood become too short for oxygen [13] and glucose [14] to be extracted by the tissue. We demonstrated that this 'physiological shunt' requires compensatory changes in blood flow to meet the metabolic needs of the tissue and, as a consequence, that tissue may be hypoxic in the absence of demonstrable signs of ischaemia [15]. In this review, we briefly describe the effects of capillary flow disturbances on oxygen and glucose extraction in tissue, and discuss whether capillary changes may contribute to the conflicting endoneurial blood flow findings in early diabetes, to the activity of abnormal metabolic pathways that contribute to diabetic complications, and more generally, to the aetiopathogenesis of glucose intolerance and diabetic complications.

\section{The relationship between tissue perfusion, capillary transit time heterogeneity and tissue oxygenation}

Historically, tissue oxygenation-defined as the maximum metabolic rate of oxygen that can be supported by the bloodstream-is inferred from the flow of oxygenated blood through the tissue. This assumption is rooted in the classic flow-diffusion equation [13], which predicts a one-to-one 
correspondence between tissue blood flow (TBF; in ml blood per $100 \mathrm{ml}$ tissue per minute) and oxygen availability $\left(\mathrm{ml} \mathrm{O}_{2}\right.$ per $100 \mathrm{ml}$ tissue per minute) when arterial blood oxygen content is at normal levels (Fig. 2). This equation assumes, however, that all tissue capillaries are equally perfused. This condition is rarely met in the tissue, where blood velocities normally vary considerably among capillaries - a phenomenon we refer to as capillary transit time heterogeneity (CTH). We recently generalised the flow-diffusion equation to express tissue oxygenation in terms of TBF, $\mathrm{CTH}$, and tissue oxygen tension $\left(\mathrm{P}_{\mathrm{t}} \mathrm{O}_{2}\right)$ [13]. For simplicity, we described the distribution of capillary transit times across the capillary bed by a realistic distribution, for which $\mathrm{CTH}$ is simply the standard deviation of capillary transit times, whereas the mean erythrocyte transit time (MTT) is given as the capillary blood volume fraction divided by TBF. Figure 2 shows tissue oxygenation for neural tissue as a function of TBF for different levels of $\mathrm{CTH}$ at constant $\mathrm{P}_{\mathrm{t}} \mathrm{O}_{2}$. In normal tissue, TBF increases are accompanied by reductions in $\mathrm{CTH}$, which limits 'physiological shunting' and maintains efficient oxygen extraction. If capillary function is impaired, however, such that $\mathrm{CTH}$ increases and capillary flows fail to homogenise during vasodilation, then increases in TBF lead to little improvement in tissue oxygenation. This phenomenon, dubbed capillary dysfunction, is the result of blood passing through capillaries at transit times too short to permit efficient extraction of its oxygen by the tissue. For capillary dysfunction with modest increases in $\mathrm{CTH}$, the poorer oxygen extraction can be compensated for by higher TBF to meet metabolic needs in tissue, and mild capillary dysfunction is therefore predicted to elicit compensatory tissue hyperaemia. If CTH increases, however, the oxygen loss due to capillary dysfunction can exceed the normal oxygenation benefits of vasodilation and hyperaemia. This paradoxical condition is termed 'malignant CTH' and is imminent if both CTH and TBF are high: in Fig. 2, this is observed at high TBF levels where oxygenation improves little with further TBF increases because physiological shunting is already high, even for negligible CTH. Therefore, flow increases must be suppressed as TBF approaches the limit of malignant $\mathrm{CTH}$ in order to avoid a paradoxical reduction in tissue oxygenation. If TBF responses are instead suppressed so that physiological shunting of blood is reduced, then the resulting fall in tissue oxygen tension (the result of ongoing cellular oxygen metabolism) will increase bloodtissue concentration gradients such that more efficient oxygen extraction can further help meet metabolic demands [13].

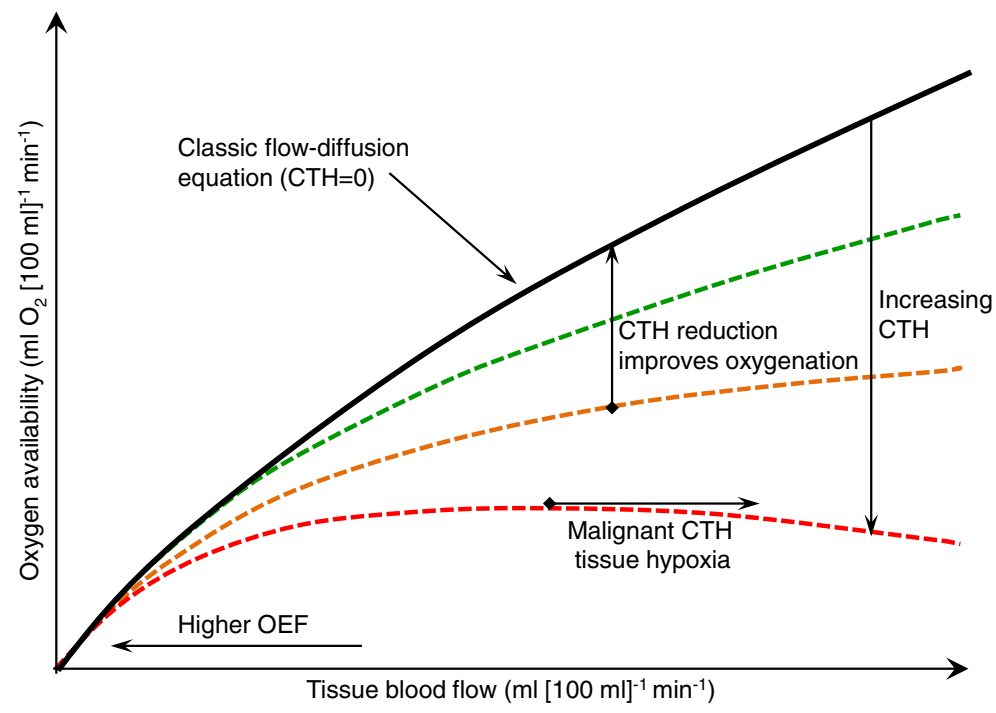

Fig. 2 The classic flow-diffusion equation (black curve) describes the relationship between TBF and the amount of a freely diffusible substance, in this case oxygen, that can be extracted by the tissue [13]. The curve is based on the extraction properties of a single capillary with blood flowing through it with a certain velocity. Note that the slope of the curve decreases with flow, indicating that the OEF decreases towards higher TBF. In generalising this relationship to tissue, it was assumed that all capillaries have identical extraction properties. Any deviation from this assumption, in the form of $\mathrm{CTH}$, reduces oxygen availability in relation to the classic flow-diffusion equation's predictions. In normal tissue, $\mathrm{CTH}$ is high during rest but is reduced during hyperaemia. Reductions in $\mathrm{CTH}$ improve oxygenation for a given TBF and thereby counteract the tendency for OEF to fall during hyperaemia. If the capillary wall is damaged or blood viscosity increased, CTH may be elevated and fail to homogenise during vasodilation. As a result, TBF increases lead to little improvements in tissue oxygenation, a phenomenon referred to as capillary dysfunction. CTH can become so high that vasodilation no longer improves tissue oxygenation - a combination of TBF and $\mathrm{CTH}$ referred to as malignant $\mathrm{CTH}$. From this point, blood flow responses must be attenuated to limit the extent of 'oxygen shunting'. Continued tissue metabolism tends to lower tissue oxygen tension, thereby increasing blood-tissue concentration gradients and oxygen extraction efficacy. The metabolic needs of nerve function can therefore be supported until the oxygen extraction fraction approaches unity and oxygen tension becomes negligible. Note that, as a result of these biophysical consequences of $\mathrm{CTH}$, both critical reductions in TBF (ischaemia) and critical increases in $\mathrm{CTH}$ (capillary dysfunction) can lead to hypoxic tissue injury. Adapted from Østergaard at al [59] 


\section{The dynamics of blood flow, blood flow responses and tissue oxygen tension as CTH increases}

Mild CTH increase: the hyperaemic state Figure 3 summarises the metabolic and haemodynamic consequences of capillary disturbances that elevate flow heterogeneity during rest, and prevent the normal flow homogenisation during hyperaemia. Elevated $\mathrm{CTH}$ reduces the maximum oxygen extraction fraction $\left(\mathrm{OEF}^{\mathrm{max}}\right)$ that can be attained for a given tissue oxygen tension [13], and the metabolic needs of tissue can therefore be met by slight increases in TBF during rest, and to some extent during activity/hyperaemic challenges. We therefore refer to states of mild CTH increases as hyperaemic.

The findings of increased sciatic blood flow $[9,10]$ after induction of diabetes by streptozotocin (STZ) in rats are therefore consistent with compensatory increases in blood flow to compensate for poorer oxygen extraction due to subtle changes in capillary flow patterns.
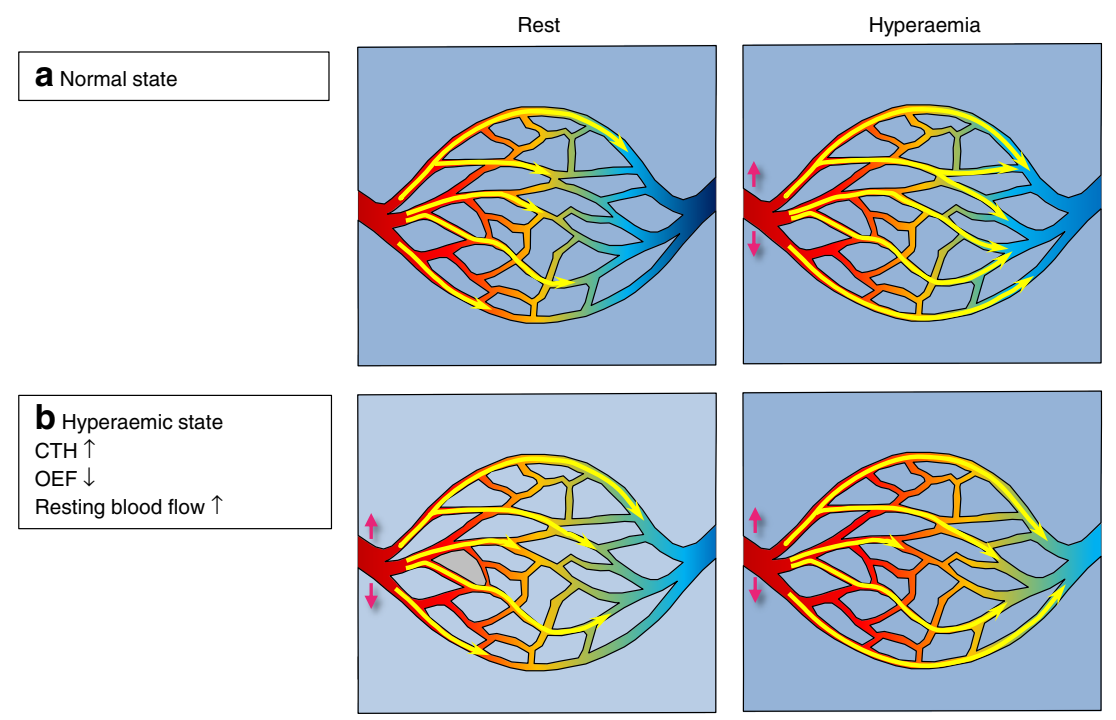

C Flow suppression state
CTH $\uparrow \uparrow$
Resting blood flow $\downarrow$
OEF $\uparrow$
Flow responses $\downarrow$
Endoneurial oxygen
tension $\downarrow$
Arteriolar tone $\uparrow$
Oxidative stress $\uparrow$
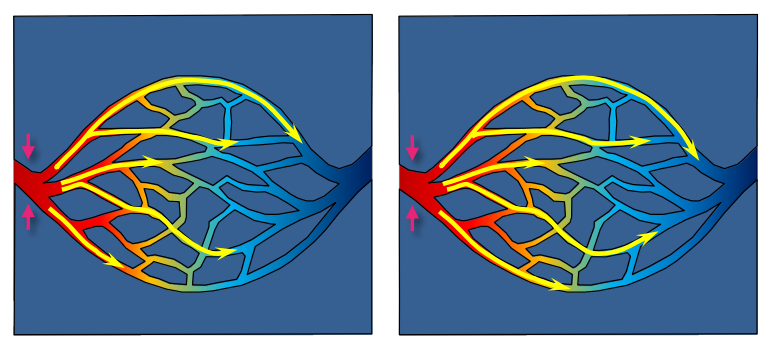
d Hypoxic state CTH $\uparrow \uparrow \uparrow$ Resting blood flow $\downarrow \downarrow$ OEF $\uparrow$
Flow responses $\downarrow \downarrow$ Endoneurial oxygen tension $\downarrow \downarrow$
Oxidative stress $\uparrow \uparrow$ Tissue damage

Fig. 3 (a) Capillary flow patterns homogenise during hyperaemia in normal tissue, counteracting the drop in OEF that would otherwise result from increased tissue blood flow (explained in Fig. 2). If CTH increases and/or fails to homogenise during functional hyperaemia, OEF is reduced. Small reductions in OEF can be compensated by higher flow and/or flow responses to meet the metabolic needs of the tissue. The hyperaemic state (b) corresponds to increases in CTH that can still be compensated for by elevated flow and/or flow responses, while the flow suppression state (c) corresponds to larger increases in CTH for which resting and/or activity-related flow responses must be suppressed in order to reduce the proportion of blood that passes through the capillary bed too fast to permit efficient oxygen extraction, and to permit the lower tissue oxygen tension (indicated by a darker blue background) to improve blood-tissue concentration gradients, and hence OEF. The suppression of flow responses (endothelial dysfunction) and low tissue oxygen tension is associated with oxidative stress and tissue inflammation. As CTH increases further and oxygen availability and tissue oxygen tension become critically low (dark blue background), the metabolic needs of normal nerve conduction can no longer be met, and nerve function becomes impaired (d). The degree of hypoxia in this state is thus predicted to reflect the degree of metabolic impairment and the severity of diabetic neuropathy. Modified from Østergaard et al [59] 
Moderate CTH increase: the flow suppression state As changes in capillary morphology or blood rheology accumulate and $\mathrm{CTH}$ increases further, increases in TBF can no longer compensate for the parallel reduction in $\mathrm{OEF}^{\max }$. Instead, TBF must be suppressed to meet the metabolic demands of neural tissue [13]. The blood supply in peripheral nerves originates from two blood supplies: an extrinsic, regional vascular system of small arteries and arterioles that connect to epineurial vessels, and a longitudinal, intrinsic system characterised by relatively wide endoneurial capillaries [16]. The two systems are interconnected by numerous epineurial and perineurial collaterals that confer considerable resistance to ischaemic damage - see Low et al for a comprehensive overview of peripheral nerve blood flow and metabolism and their relation to nerve damage under diabetic and ischaemic conditions [16]. The tone of epineurial arteries and arterioles is affected by dense perivascular plexuses of noradrenergic, serotonergic and peptidergic nerve fibres, while the intrinsic arterioles display a relative lack of vascular smooth muscle cells [16]. Any reductions in flow or flow responses in relation to increasing capillary dysfunction would therefore be expected to involve epineurial microvessels.

Suppression of endoneurial blood flow and impaired relaxation of epineurial resistance vessels in response to standardised vasodilatory stimuli, so-called endothelial dysfunction, is observed in STZ-induced diabetes in rats prior to any reductions in motor nerve conduction velocity (MNCV) and $\mathrm{Na}^{+} / \mathrm{K}^{+}$ATPase [17]. Indeed, endoneurial oxygen tension has been observed to decrease prior to the decrease in neural blood flow and the onset of neuropathy in STZ-induced diabetes [18], consistent with the prediction that flow suppression represents a compensatory mechanism to ensure sufficient oxygen extraction, rather than the primary cause of nerve dysfunction. Endothelial dysfunction is associated with increased production of reactive oxygen species (ROS) in the vessel wall, and with parallel depletion of the vasodilator NO as it reacts with ROS to produce peroxynitrite [19]. However, oxidative stress [20] and NO depletion [21] are also powerful capillary constrictors. The reversal of endothelial dysfunction and nerve conduction deficits following antioxidant treatment in rats with STZ-induced diabetes [22, 23] may therefore reflect the reversal of capillary dysfunction. If capillary dysfunction is irreversible owing to permanent capillary damage, then restoration of nerve blood flow would not be expected to result in improved endoneurial oxygenation. According to this prediction, antioxidant treatment is therefore expected to be less efficacious in disease models and patients with irreversible capillary flow disturbances.

The prediction that increasing $\mathrm{CTH}$ is associated with a transition from endoneurial hyperperfusion, when capillary changes are still mild, to normo- and then hypoperfusion when capillary changes become more severe, distinguishes capillary dysfunction from a condition in which blood flow, rather than oxygen extraction, is limited by microvascular changes. Tesfaye et al measured epineurial perfusion in the sural nerve and found reduced blood flow in diabetic patients with chronic sensorimotor neuropathy compared with controls, but increased flow in diabetic patients without neuropathy [24]. These findings are therefore consistent with a progression in capillary dysfunction, with sural nerve hyperperfusion (with preserved oxygen supply-demand balance) in diabetic patients prior to the development of neuropathy, progressing to hypoperfusion (with oxidative stress and hypoxia) as their neuropathy develops.

Large CTH increase: the hypoxic state and reduced nerve conduction velocity If CTH increases even further, the parallel reduction of tissue oxygen tension can contribute to neural tissue dysfunction or damage in several ways. First, the lack of oxygen, and thus of ATP to fuel neural functions, is likely to cause tissue dysfunction. Second, a reduction in tissue oxygen tension upregulates the expression of hypoxia inducible factor 1 (HIF-1) and nuclear factor- $\mathrm{KB}(\mathrm{NF}-\mathrm{kB})$, both of which are strong pro-inflammatory signals [25]. Indeed, NF- $k B$ levels are elevated in peripheral nerves and dorsal root ganglia in experimental diabetic neuropathy [26], and in humans, both central and peripheral levels of inflammatory markers correlate with the severity of DNP [27]. Third, HIF-1 also upregulates levels of NADPH oxidase 2 (NOX-2) levels [28], a major source of ROS in endothelial dysfunction [29]. ROS in turn react with NO to produce peroxynitrite [19], a source of severe nitrosative tissue damage. In addition, peroxynitrite inactivates tissue plasminogen activator (tPA), consistent with the lack of detectable tPA in endo- and epineurial vessels in patients with diabetic neuropathy [30]. In neuronal tissue, tPA levels determine the formation of brain-derived neurotrophic factor (BDNF) from its precursor, proBDNF. Whereas BDNF is known to provide trophic support for neurons and astrocytes, proBDNF induces neuronal apoptosis [31]. The reduction in distal muscle BDNF and nerve growth factor (NGF) levels indeed correlate with the severity of neuropathy in diabetic patients [32]. This pathway mediates neurodegeneration in diabetic neuropathy [33] and provides a mechanism by which gradual reductions in oxygen availability cause a gradual shift from a state of trophic support for neuronal survival and function to a state of gradual reduction in neuronal fibre number to better match oxygen availability. The relationships between pro-neurotrophins and nociception are discussed in Richner et al [34].

The prediction that nerve fibre function may be supported until the stage where low tissue oxygen tension can no longer secure sufficient oxygen extraction is consistent with the finding that reductions in sural nerve sensory conduction velocity correlate better with nerve oxygen tension than with blood flow values in patients with diabetic neuropathy $[35,36]$. 
Intuitively, one might expect tissue hypoxia to elicit angiogenesis and hence the formation of new capillaries to improve tissue oxygenation. Such capillaries would, however, tend to become immediate shunts for blood that would otherwise pass through capillaries with higher resistance, yet more efficient oxygen extraction [14] (see Fig. 4). Angiogenesis may therefore, paradoxically, exacerbate tissue hypoxia in conditions with pre-existing capillary damage, consistent with reports that insulin neuritis is associated with epineurial microvascular proliferation and excessive arteriovenous shunting [37]. Pericytes are crucial in the initiation of angiogenesis [38]; we speculate that pericyte dysfunction further limits angiogenesis in diabetes.

\section{ATP production when $\mathrm{CTH}$ is elevated: differential effects of capillary dysfunction on oxygen and glucose extraction}

Glucose and oxygen are the predominant substrates for the production of the ATP needed for normal peripheral nerve function [39]. The uptake of glucose into the endoneurium is not believed to be insulin dependent [39], but the blood-nerve barrier itself appears to limit the endoneurial extraction of glucose analogues [40]. Unlike rats, humans have few GLUT-1 proteins in endoneurial capillaries [41]. Endoneurial glucose extraction is therefore thought to be limited by the integrity of the blood-nerve barrier rather than by the kinetic properties of glucose transporters [41], and therefore to depend on $\mathrm{CTH}$ in much the same way as oxygen extraction. Indeed, indicator dilution studies in the brain show that the extraction of glucose and glucose analogues by the central nervous system is limited by $\mathrm{CTH}$, and that efficient glucose extraction during hyperaemia depends on homogenisation of capillary transit times [42], as discussed for oxygen above. Using these characteristics, the ratio between glucose and oxygen extraction in neural tissue can be assessed, based on the assumption that endoneurial capillaries display a tenfold higher capillary permeability to glucose than those of the brain [40]. This may still be a conservative estimate in that the blood-nerve barrier integrity is disturbed in diabetes, increasing its permeability to glucose and glycosylated serum proteins [43]. Figure 4 shows the ratio between glucose and oxygen extraction as a function of MTT and CTH under these assumptions. Note that, as $\mathrm{CTH}$ increases, this ratio is reduced because oxygen uptake is hindered more by $\mathrm{CTH}$ and oxygen's binding to haemoglobin than is glucose. Below, we briefly discuss this aspect of capillary dysfunction in relation to the ATP needs of peripheral nerve function.

Under aerobic conditions, glucose metabolism by oxidative phosphorylation generates 29-30 ATP molecules per molecule of glucose, but when oxygen availability is limited,

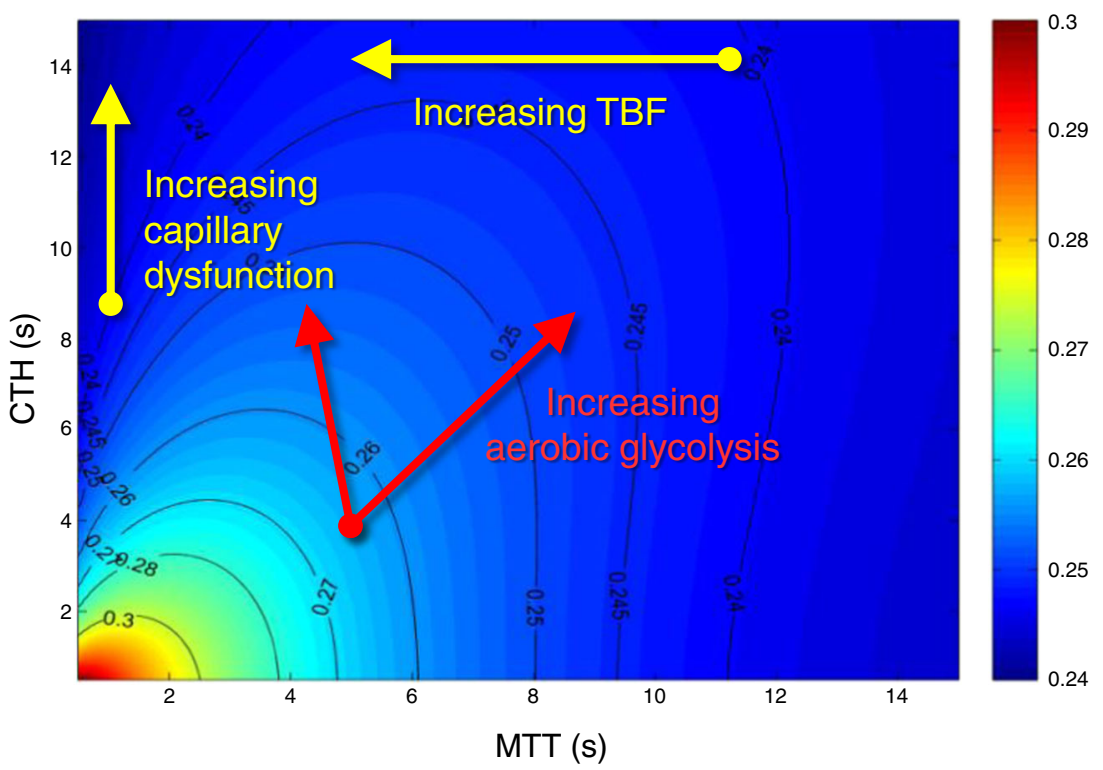

Fig. 4 Contour plot showing the relationship between the capillary MTT as blood flows through tissue ( $x$-axis), its $\mathrm{CTH}$ along the $y$-axis, and the ratio between the net extraction of oxygen and glucose, respectively, as indicated by a colour scale. Warm colours correspond to a high ratio, which permits oxidative phosphorylation to predominate, while blue colours correspond to aerobic glycolysis with limited ATP yields. MTT is defined as the capillary blood volume divided by blood flow, and angiogenesis (which increases capillary density) therefore increases MTT unless blood flow increases in parallel with capillary density.
Diabetic angioproliferation tends to produce chaotic microvessels with multiple shunts [37] and would therefore be expected to cause an increase in CTH. The red arrows illustrate two instances of elevated $\mathrm{CTH}-$ one in which blood flow increased in parallel with capillary density (left), and one in which upstream microvascular changes prevented TBF changes (right). In both cases, the differential extraction of oxygen and glucose favours lactate formation rather than oxidative phosphorylation. The black lines indicate iso-contours, for which the oxygen:glucose extraction ratio is given by numbers. Adapted from Østergaard et al [14] 
glucose undergoes anaerobic glycolysis, forming two lactate molecules with an ATP yield of only two - down by a factor of 15 compared with oxidative phosphorylation. In the eye, the kidney, and the myelin sheaths of peripheral nerves, aldose reductase enzymes are present, allowing the conversion of glucose into sorbitol without using the two ATP molecules that are required during the initial phosphorylation step of oxidative phosphorylation. The aldose reductase pathway can therefore preserve endoneurial ATP when oxygen extraction is limited by capillary dysfunction, in that it reduces the ATP expenditure needed to maintain energy-efficient oxidative phosphorylation. However, capillary dysfunction is also predicted to reduce glucose extraction, making less glucose available for the aldose reductase pathway. Thus, ATP production via this pathway may be insufficient to secure normal peripheral nerve function. The possible links between nerve energy status (hypoxia, low $\mathrm{pH}$ and increased lactate levels) and pain are discussed in more detail below.

We note that, according to this prediction, increased utilisation of the aldose reductase pathway in diabetes is a result of increasing capillary damage, rather than of high blood glucose per se. In particular, inhibition of fructose formation via this pathway would be expected to worsen the energy crisis of peripheral nerves in diabetes by favouring glucose metabolism via the more ATP-demanding phosphorylation pathway. This is consistent with findings that sorbitol dehydrogenase inhibitors fail to increase nerve blood flow or conduction velocity in experimental diabetes [44] but instead worsen nerve energy status in some studies [45], and apparently exacerbate sympathetic autonomic neuropathy in STZinduced diabetes [46].

Despite its ability to preserve ATP production for nerve function, activation of the aldose reductase pathway is known to have deleterious long-term effects: the conversion of glucose into sorbitol uses NADPH, and the subsequent conversion of sorbitol into fructose uses NAD, both of which alter the cell redox state [47]. In particular, NADPH is important for the regeneration of reduced glutathione, an important reactive oxygen species (ROS) scavenger. Long-term activation of the aldose reductase pathway is therefore expected to cause oxidative damage to peripheral nerves [47]. Aldose reductase pathway inhibition is therefore potentially a double-edged sword in that it reduces oxidative stress on the one hand, while exacerbating tissue energy crisis on the other by causing a shift to less ATP-efficient glucose metabolism. We speculate that these effects may explain why aldose reductase inhibitors have attenuated the progression of neuropathic changes in some clinical trials, while failing to do so in others [48, 49].

Capillary damage and oxidative stress due to hyperglycaemia Hyperglycaemia causes the formation of AGEs via non-enzymatic reactions between aldehyde groups of reducing sugars with proteins, lipids and nucleic acids. The production of AGEs is associated with ROS production, just as AGEs interact with the AGE receptor (RAGE) causing further ROS release [29, 47], and consequently oxidative damage.

Hyperglycaemia [50], oxidative stress and oxidised lipoproteins [51, 52] disrupt the glycocalyx, a $0.5 \mu \mathrm{m}$ thick carbohydrate-rich matrix that covers the luminal surface of the capillary endothelium [53]. The glycocalyx is thought to play a key role in the control of erythrocyte flow through the capillary bed [54], and its disruption is therefore likely to cause capillary dysfunction. Underscoring this regulatory role, glycocalyx disruption causes an increase in capillary haematocrit from only $20-50 \%$ of full blood haematocrit, to approach values similar to those found in the systemic circulation [52]. Mice fed a high-fat diet to generate high levels of oxidised lipoprotein and oxidative stress develop reduced nerve conduction velocities and sensory deficits before glucose tolerance is impaired [55]. This finding is consistent with a role of generalised capillary dysfunction in the development of glucose intolerance, alongside the development of reduced nerve conduction velocity as a result of endoneurial capillary dysfunction and hypoxia; see below.

\section{Sources of pain in diabetic neuropathy}

While progressive changes in capillary morphology and function may cause reductions in nerve function and even nerve damage, these mechanisms fail to explain the mechanical hyperalgesia and tactile allodynia (sensation of pain in response to otherwise non-painful stimuli) experienced by nearly half of patients with diabetic neuropathy [3]. Endoneurial hypoxia is associated with upregulation of HIF-1 and NF- KB [25] (see above). NF-kB is crucial in the regulation of developmental and synaptic plasticity and can prevent the death of neurons by the production of anti-apoptotic proteins [56]. While the activation of NF-KB may thus serve to protect nerve integrity and function under conditions of hypoxia, it also appears to be involved in neuropathic and inflammatory pain [57]. Notably, sulfasalazine reduces the expression of NF- $\mathrm{KB}$ p50 in both sciatic nerves and dorsal root ganglia of STZ diabetic rats, blocking their development of tactile allodynia [58]. The relationships between pro-neurotrophin levels (above) and nociception are discussed in detail elsewhere [34], and the relation between tissue injury, tissue hypoxia and pain, in Østergaard et al [59].

Painful diabetic neuropathy may be related to the function of small, autonomic fibres in diabetic patients. Small fibre dysfunction with sympathetic denervation of the peripheral arterial system is thought to occur quite early in the progression of neuropathy [60]. The resulting loss of vasoconstrictor tone and peripheral vasodilatation gives rise to the appearance 
of a warm, oedematous neuropathic foot [60]. The high peripheral blood flow passes through arteriovenous shunts [60], and it was recently hypothesised that excessive microvascular shunting may give rise to tissue hypoxia, despite the high blood flow [61]. Our model of oxygen extraction in tissue [13] supports this notion, and predicts that failure to suppress blood flow in capillary dysfunction can be the source of severe oxidative stress, microvascular injury and pain [59]. Archer et al [62] showed that blood flow in the feet of patients with diabetic neuropathy is five times higher than in normal controls. While patients with painful diabetic neuropathy had slightly lower blood flow than those without pain, the groups differed further by the preserved ability of sympathetic stimuli to suppress blood flow in the group with painful diabetic neuropathy. Furthermore, reductions in blood flow were associated with a reduction in neuropathic pain, similar to the pain relief reported by some patients when cooling the feet (which would be expected to cause local vasoconstriction) [62]. Taken together, these observations support the role of hypoxia in painful diabetic neuropathy and suggest that small sympathetic fibres play a role in the pain mechanism, possibly in relation to their vasomotor action under conditions where suppression of peripheral blood flow appears important to meet the metabolic needs of the tissue.

\section{The role of capillary pericytes in diabetic complications}

Loss of pericytes is evident in biopsy material from patients with diabetic neuropathy [4], and pericyte loss is closely related to the severity of diabetic retinopathy $[63,64]$. In the central nervous system [65, 66] and the retina [67-69], pericytes regulate capillary diameter according to local metabolic needs. Pericytes and endothelial cells form the capillary basal membrane [38], which (in addition to those of peripheral nerves) is thickened in several organs in diabetes [70, 71]. Based on their proposed role in maintaining efficient oxygen extraction, means of supporting pericyte function and survival might therefore be expected to alleviate diabetic neuropathy. This notion is in agreement with animal models of diabetic retinopathy, where the development of retinal damage appears to be closely related to pericyte apoptosis [64]. Of note, the rescue of retinal pericytes was recently shown to prevent diabetic retinopathy in animal models [64].

The control of pericyte tone remains much less studied than that of arteriolar tone [72]. Studies of retinal capillaries suggest that pericytes react to intrinsic signalling in much the same way as smooth muscle cells. Pericyte constriction has been observed in response to mechanical stretch, exposure to angiotensin II (via $\mathrm{AT}_{1}$ receptors) [73], and endothelin-1 (via $\mathrm{ET}_{\mathrm{A}}$ receptors) [74], by a $\mathrm{Ca}^{2+}$ dependent mechanism [75]. Retinal pericytes relax in response to $\mathrm{NO}[21]$ and adrenergic (via $\beta_{2}$ receptors) [75] stimulation. In cerebral pericytes, ischaemia and oxidative stress cause irreversible capillary constriction $[20,66]$.

Restoration of capillary NO levels would be expected to improve CTH (homogenising capillary flow patterns) by facilitating pericyte relaxation. This may be achieved in ways that do not require oxygen as a substrate for NO synthesis, namely by dietary administration of nitrate or nitrite, which is readily converted to NO in the tissue [76]. Green leafy vegetables are sources of dietary nitrate and seemingly reduce the risk of developing type 2 diabetes [77]. Meanwhile, topical application of nitrate reduces neuropathic pain and burning sensation, but not other sensory modalities, in patients with painful diabetic neuropathy [78]. Pharmacologically, antihypertensive drugs would be expected to modulate the effects of angiotensin and endothelin on pericyte tone, or its $\mathrm{Ca}^{2+}$ dependent regulation. ACE inhibitor treatment has been shown to improve nerve conduction, but not autonomic function, vibration perception threshold, or neuropathy symptom and deficit score, in normotensive diabetic patients [79]. Furthermore, ACE inhibitor and angiotensin II receptor antagonist treatment improved nerve conduction velocities, reduced oxidative stress, and reverted endoneurial flow suppression in STZ mice [80]. Interestingly, ACE inhibitor administration prior to the induction of diabetes by STZ in rats was found to prevent development of nerve conduction abnormalities [81]. Calcium blocker treatment has also been reported to reverse flow suppression in the vasa nervorum of STZ diabetic rats [82], and to improve their motorand sensory nerve conduction velocity [83].

\section{Potential implications for diabetes management}

Our review suggests that early loss of capillary flow control and changes in capillary morphology may play a central role in the aetiopathogenesis of diabetic neuropathy.

The prediction that elevated CTH impairs both oxygen and glucose extraction in tissue also implies that strategies to prevent diabetic complications may differ between patients with type 1 and type 2 diabetes, respectively. The diagnosis of type 1 diabetes marks the onset of hyperglycaemia-related capillary damage to peripheral nerves (as explained above) and organ microvasculature in general, to an extent that would be expected to depend on the cumulative exposure to hyperglycaemia. Early, intensive glycaemic control indeed delays the onset of type 1 diabetes complications, including diabetic neuropathy [84]. By contrast, type 2 diabetes and its associated complications, risk factors such as age, obesity and hypertension, are all associated with either degenerative changes in capillary morphology [85] or dysfunctional angiogenesis [86] prior to the onset of type 2 diabetes. Indeed, given 
the effects of CTH on glucose clearance from blood, the progressive capillary dysfunction of the systemic microcirculation caused by type 2 diabetes risk factors is likely to reduce glucose tolerance, and hence contribute to what we define as type 2 diabetes. We therefore suggest that type 2 diabetes complications represent the progression of systemic capillary dysfunction from more moderate levels already present when type 2 diabetes is diagnosed. This is consistent with recent observations of early small fibre loss in the cornea of patients with impaired glucose tolerance [87] and recently diagnosed type 2 diabetes [88], keeping in mind that while the cornea is avascular, the proximal course of its fibres depend on capillary function to maintain function and trophic support.

The prediction that hyperglycaemia is one of many sources of capillary dysfunction in type 2 diabetes suggests that its comorbidities and risk factors, including hypertension, systemic inflammation, hypercholesterolaemia and smoking, should be viewed and managed as separate, modifiable sources of additional capillary dysfunction. (1) In angiotensin II models of hypertension, flow responses are indeed attenuated in some organs prior to the development of increased blood pressure [89], suggesting that the increased peripheral resistance in hypertension represents a systemic response to preserve tissue oxygenation in response to widespread capillary/pericyte constrictions and elevated CTH in response to this powerful pericyte constrictor. (2) Animal studies of systemic inflammation have shown that capillary flow patterns are sensitive to the size, viscosity, number and endothelial adhesion of blood cells, and undergo profound changes as part of the low-grade vascular inflammation that accompanies many cardiovascular risk factors $[51,90]$. In diabetic patients, blood viscosity at low shear rates is indeed elevated, correlating with the extent of their microvascular diabetic complications [91]. See also a discussion of blood viscosity changes in diabetes in Low et al [16]. (3) Plasma lipid levels also affect blood viscosity, and high triacylglycerol and cholesterol levels are therefore predicted to represent an independent risk factor for type 2 diabetes and its complications, while lipid-lowering therapy would be predicted to reduce CTH and hence improve endoneurial oxygenation while reducing oxidative damage and the development and progression of diabetic neuropathy. This is consistent with observations that triacylglycerol levels correlate with the progression of diabetic neuropathy [92], with clinical trials [93], cohort studies [94, 95] showing benefits of statin treatment in type 2 diabetes, and with animal studies showing restoration of vasa nervorum function and reversal of diabetic neuropathy after statin treatment [96]. Importantly, fibrates (which lower triacylglycerol and cholesterol levels) and statins seem more efficient than intensive blood glucose control in reducing the rate of amputation in type 2 diabetes [97]. While plasma viscosity may represent a putative target for diabetes management, we propose that observations of neuropathic pain severity during infections, where leucocytosis causes capillary flow patterns to become more disturbed [90], would serve as an indirect confirmation of the role of capillary dysfunction in diabetic neuropathy. (4) Nicotine upregulates the expression of adhesion molecules in the capillary endothelium [98] and increases leucocyte rolling [99], consistent with findings that smoking represents an independent risk factor for diabetic neuropathy [100]. Cessation of nicotine exposure would therefore be predicted to alleviate both symptoms and progression of diabetic neuropathy. Similarly, high homocysteine levels increase blood viscosity and the adhesion of monocytes to the capillary wall, and increase the oxidation of low-density lipoproteins [101] (see the section on glycocalyx function above). These effects would be expected to cause capillary dysfunction and progression of neuropathy, consistent with reports that homocysteine is independently associated with diabetic neuropathy in patients with type 2 diabetes [102]. Similarly, findings of more severe neuropathy in type 2 diabetic patients who had received metformin may be related to the accompanying increases in blood homocysteine levels, in addition to the effects of longterm reduction in cobalamine (vitamin $\mathrm{B}_{12}$ ) levels [103].

\section{Conclusion}

The proposed hypothesis that capillary dysfunction causes diabetic neuropathy (and some degree of glucose intolerance) gives rise to a range of predictions that lend themselves to further scrutiny in animal experiments, epidemiological studies and clinical trials. The hypothesis relates type 2 diabetes risk factors, and effects of poor glycaemic control on capillary function in both type 1 and type 2 diabetes, to their effects on blood rheology and the morphology and function of capillaries. Accordingly, we predict that animal models of diabetic complications should display capillary dysfunction or damage similar to that observed in human nerves, kidney and retina in order to predict the translational potential of experimental therapies. Pericyte function and pericyte damage also appear to be important to our understanding of diabetic neuropathy.

Capillary dysfunction is summarised in a single parameter, $\mathrm{CTH}$, which we propose determines the derived effects on extraction of oxygen and glucose in various tissue types. So far, studies of diabetic neuropathy have focused on nerve blood flow rather than its capillary distribution. To extend the indirect evidence of capillary dysfunction presented here, capillary flow velocities [104] and nerve oxygen tension [105] must therefore be imaged longitudinally and related to nerve function in animal models of diabetes. To translate such studies into human disease, microvascular flow distributions and $\mathrm{CTH}$ may be estimated noninvasively by dynamic tracking of intravascular contrast agent retention after bolus 
injection [106], using, for example, contrast enhanced ultrasound to capture the haemodynamics in peripheral nerves.

Funding This study was supported by the Danish National Research Foundation (Center of Functionally Integrative Neuroscience; LØ, RAO and SNJ), the Danish Ministry of Science, Innovation, and Education (MINDLab; LØ, NBF, RAO, KRD SNJ and TSJ), and the VELUX Foundation (Aarhus Research Center for Brain Aging and Dementia [ARCADIA]; LØ and SNJ).

Duality of interest The authors declare that there is no duality of interest associated with this manuscript.

Contribution statement LØ conceived the study and wrote the first draft. SNJ developed the biophysical model and contour plot. All authors conducted literature searches and made substantial contributions to the interpretation of literature data in relation to the study hypotheses. All authors revised the manuscript for important intellectual content and approved the final version.

Open Access This article is distributed under the terms of the Creative Commons Attribution License which permits any use, distribution, and reproduction in any medium, provided the original author(s) and the source are credited.

\section{References}

1. Callaghan BC, Cheng HT, Stables CL, Smith AL, Feldman EL (2012) Diabetic neuropathy: clinical manifestations and current treatments. Lancet Neurol 11:521-534

2. Peltier A, Goutman SA, Callaghan BC (2014) Painful diabetic neuropathy. BMJ 348:g1799

3. Tesfaye S, Selvarajah D (2012) Advances in the epidemiology, pathogenesis and management of diabetic peripheral neuropathy. Diabetes Metab Res Rev 28(Suppl 1):8-14

4. Giannini C, Dyck PJ (1995) Basement membrane reduplication and pericyte degeneration precede development of diabetic polyneuropathy and are associated with its severity. Ann Neurol 37:498-504

5. Thrainsdottir S, Malik RA, Dahlin LB et al (2003) Endoneurial capillary abnormalities presage deterioration of glucose tolerance and accompany peripheral neuropathy in man. Diabetes 52:26152622

6. Cameron NE, Eaton SE, Cotter MA, Tesfaye S (2001) Vascular factors and metabolic interactions in the pathogenesis of diabetic neuropathy. Diabetologia 44:1973-1988

7. Tuck RR, Schmelzer JD, Low PA (1984) Endoneurial blood flow and oxygen tension in the sciatic nerves of rats with experimental diabetic neuropathy. Brain 107:935-950

8. Jaap AJ, Hammersley MS, Shore AC, Tooke JE (1994) Reduced microvascular hyperaemia in subjects at risk of developing type 2 (non-insulin-dependent) diabetes mellitus. Diabetologia 37:214 216

9. Tilton RG, Chang K, Hasan KS et al (1993) Prevention of diabetic vascular dysfunction by guanidines. Inhibition of nitric oxide synthase versus advanced glycation end-product formation. Diabetes 42:221-232

10. Tilton RG, Chang K, Nyengaard JR, van den Enden M, Ido Y, Williamson JR (1995) Inhibition of sorbitol dehydrogenase. Effects on vascular and neural dysfunction in streptozocin-induced diabetic rats. Diabetes 44:234-242
11. Theriault M, Dort J, Sutherland G, Zochodne DW (1997) Local human sural nerve blood flow in diabetic and other polyneuropathies. Brain 120:1131-1138

12. Vincent AM, Calabek B, Roberts L, Feldman EL (2013) Biology of diabetic neuropathy. Handb Clin Neurol 115:591-606

13. Jespersen SN, Østergaard L (2012) The roles of cerebral blood flow, capillary transit time heterogeneity and oxygen tension in brain oxygenation and metabolism. J Cereb Blood Flow Metab 32:264 277

14. Østergaard L, Tietze A, Nielsen T et al (2013) The relationship between tumor blood flow, angiogenesis, tumor hypoxia, and aerobic glycolysis. Cancer Res 73:5618-5624

15. Østergaard L, Aamand R, Gutierrez-Jimenez E et al (2013) The capillary dysfunction hypothesis of Alzheimer's disease. Neurobiol Aging 34:1018-1031

16. Low PA, Lagerlund TD, McManis PG (1989) Nerve blood flow and oxygen delivery in normal, diabetic, and ischemic neuropathy. Int Rev Neurobiol 31:355-438

17. Coppey LJ, Davidson EP, Dunlap JA, Lund DD, Yorek MA (2000) Slowing of motor nerve conduction velocity in streptozotocininduced diabetic rats is preceded by impaired vasodilation in arterioles that overlie the sciatic nerve. Int J Exp Diabetes Res 1:131-143

18. Zochodne DW, Ho LT (1992) Normal blood flow but lower oxygen tension in diabetes of young rats: microenvironment and the influence of sympathectomy. Can J Physiol Pharmacol 70:651-659

19. Obrosova IG, Drel VR, Oltman CL et al (2007) Role of nitrosative stress in early neuropathy and vascular dysfunction in streptozotocin-diabetic rats. Am J Physiol Endocrinol Metab 293: E1645-E1655

20. Yemisci M, Gursoy-Ozdemir Y, Vural A, Can A, Topalkara K, Dalkara T (2009) Pericyte contraction induced by oxidativenitrative stress impairs capillary reflow despite successful opening of an occluded cerebral artery. Nat Med 15:1031-1037

21. Haefliger IO, Zschauer A, Anderson DR (1994) Relaxation of retinal pericyte contractile tone through the nitric oxide-cyclic guanosine monophosphate pathway. Invest Ophthalmol Vis Sci 35: 991-997

22. Cameron NE, Cotter MA, Archibald V, Dines KC, Maxfield EK (1994) Anti-oxidant and pro-oxidant effects on nerve conduction velocity, endoneurial blood flow and oxygen tension in non-diabetic and streptozotocin-diabetic rats. Diabetologia 37:449-459

23. Coppey LJ, Gellett JS, Davidson EP, Dunlap JA, Lund DD, Yorek MA (2001) Effect of antioxidant treatment of streptozotocininduced diabetic rats on endoneurial blood flow, motor nerve conduction velocity, and vascular reactivity of epineurial arterioles of the sciatic nerve. Diabetes 50:1927-1937

24. Tesfaye S, Harris N, Jakubowski JJ et al (1993) Impaired blood flow and arterio-venous shunting in human diabetic neuropathy: a novel technique of nerve photography and fluorescein angiography. Diabetologia 36:1266-1274

25. Eltzschig HK, Carmeliet P (2011) Hypoxia and inflammation. N Engl J Med 364:656-665

26. Toth C, Rong LL, Yang C et al (2008) Receptor for advanced glycation end products (RAGEs) and experimental diabetic neuropathy. Diabetes 57:1002-1017

27. Kallestrup M, Moller HJ, Tankisi H, Andersen H (2014) Soluble CD163 levels are elevated in cerebrospinal fluid and serum in people with type 2 diabetes mellitus and are associated with impaired peripheral nerve function. Diabet Med. doi:10.1111/dme.12568

28. Yuan G, Khan SA, Luo W, Nanduri J, Semenza GL, Prabhakar NR (2011) Hypoxia-inducible factor 1 mediates increased expression of NADPH oxidase-2 in response to intermittent hypoxia. J Cell Physiol 226:2925-2933

29. Pop-Busui R, Sima A, Stevens M (2006) Diabetic neuropathy and oxidative stress. Diabetes Metab Res Rev 22:257-273 
30. Hafer-Macko CE, Ivey FM, Sorkin JD, Macko RF (2007) Microvascular tissue plasminogen activator is reduced in diabetic neuropathy. Neurology 69:268-274

31. Teng HK, Teng KK, Lee R et al (2005) ProBDNF induces neuronal apoptosis via activation of a receptor complex of p75NTR and sortilin. J Neurosci 25:5455-5463

32. Andreassen CS, Jakobsen J, Flyvbjerg A, Andersen H (2009) Expression of neurotrophic factors in diabetic muscle-relation to neuropathy and muscle strength. Brain 132:2724-2733

33. Ali TK, Matragoon S, Pillai BA, Liou GI, El-Remessy AB (2008) Peroxynitrite mediates retinal neurodegeneration by inhibiting nerve growth factor survival signaling in experimental and human diabetes. Diabetes 57:889-898

34. Richner M, Ulrichsen M, Elmegaard SL, Dieu R, Pallesen LT, Vaegter CB (2014) Peripheral nerve injury modulates neurotrophin signaling in the peripheral and central nervous system. Mol Neurobiol 50:945-970

35. Ibrahim S, Harris ND, Radatz M et al (1999) A new minimally invasive technique to show nerve ischaemia in diabetic neuropathy. Diabetologia 42:737-742

36. Young MJ, Veves A, Walker MG, Boulton AJ (1992) Correlations between nerve function and tissue oxygenation in diabetic patients: further clues to the aetiology of diabetic neuropathy? Diabetologia $35: 1146-1150$

37. Tesfaye S, Malik R, Harris N et al (1996) Arterio-venous shunting and proliferating new vessels in acute painful neuropathy of rapid glycaemic control (insulin neuritis). Diabetologia 39:329-335

38. Armulik A, Abramsson A, Betsholtz C (2005) Endothelial/pericyte interactions. Circ Res 97:512-523

39. Greene DA, Winegrad AI (1979) In vitro studies of the substrates for energy production and the effects of insulin on glucose utilization in the neural components of peripheral nerve. Diabetes 28:878-887

40. Rechthand E, Smith QR, Rapoport SI (1989) Structural specificity of sugar transport at the blood-nerve barrier. J Neurochem 53:119123

41. Muona P, Jaakkola S, Salonen V, Peltonen J (1993) Expression of glucose transporter 1 in adult and developing human peripheral nerve. Diabetologia 36:133-140

42. Knudsen GM, Pettigrew KD, Paulson OB, Hertz MM, Patlak CS (1990) Kinetic analysis of blood-brain barrier transport of D-glucose in man: quantitative evaluation in the presence of tracer backflux and capillary heterogeneity. Microvasc Res 39:28-49

43. Poduslo JF, Curran GL (1992) Increased permeability across the blood-nerve barrier of albumin glycated in vitro and in vivo from patients with diabetic polyneuropathy. Proc Natl Acad Sci U S A 89: 2218-2222

44. Cameron NE, Cotter MA, Basso M, Hohman TC (1997) Comparison of the effects of inhibitors of aldose reductase and sorbitol dehydrogenase on neurovascular function, nerve conduction and tissue polyol pathway metabolites in streptozotocindiabetic rats. Diabetologia 40:271-281

45. Obrosova IG, Fathallah L, Lang HJ, Greene DA (1999) Evaluation of a sorbitol dehydrogenase inhibitor on diabetic peripheral nerve metabolism: a prevention study. Diabetologia 42:1187-1194

46. Schmidt RE, Dorsey DA, Beaudet LN et al (2005) A potent sorbitol dehydrogenase inhibitor exacerbates sympathetic autonomic neuropathy in rats with streptozotocin-induced diabetes. Exp Neurol 192:407-419

47. Sytze van Dam P, Cotter MA, Bravenboer B, Cameron NE (2013) Pathogenesis of diabetic neuropathy: focus on neurovascular mechanisms. Eur J Pharmacol 719:180-186

48. Hotta N, Akanuma Y, Kawamori R et al (2006) Long-term clinical effects of epalrestat, an aldose reductase inhibitor, on diabetic peripheral neuropathy: the 3-year, multicenter, comparative Aldose Reductase Inhibitor-Diabetes Complications Trial. Diabetes Care 29:1538-1544
49. Bril V, Hirose T, Tomioka S, Buchanan R, Ranirestat Study Group (2009) Ranirestat for the management of diabetic sensorimotor polyneuropathy. Diabetes Care 32:1256-1260

50. Nieuwdorp M, Mooij HL, Kroon J et al (2006) Endothelial glycocalyx damage coincides with microalbuminuria in type 1 diabetes. Diabetes 55:1127-1132

51. Vink H, Constantinescu AA, Spaan JA (2000) Oxidized lipoproteins degrade the endothelial surface layer: implications for plateletendothelial cell adhesion. Circulation 101:1500-1502

52. Constantinescu AA, Vink H, Spaan JA (2001) Elevated capillary tube hematocrit reflects degradation of endothelial cell glycocalyx by oxidized LDL. Am J Physiol Heart Circ Physiol 280:H1051H1057

53. Vink H, Duling BR (1996) Identification of distinct luminal domains for macromolecules, erythrocytes, and leukocytes within mammalian capillaries. Circ Res 79:581-589

54. Secomb TW, Hsu R, Pries AR (1998) A model for red blood cell motion in glycocalyx-lined capillaries. Am J Physiol 274:H1016 H1022

55. Vincent AM, Hayes JM, McLean LL, Vivekanandan-Giri A, Pennathur S, Feldman EL (2009) Dyslipidemia-induced neuropathy in mice: the role of oxLDL/LOX-1. Diabetes 58:2376-2385

56. Mattson MP, Meffert MK (2006) Roles for NF-kB in nerve cell survival, plasticity, and disease. Cell Death Differ 13:852-860

57. Tegeder I, Niederberger E, Schmidt R et al (2004) Specific inhibition of IKB kinase reduces hyperalgesia in inflammatory and neuropathic pain models in rats. J Neurosci 24:1637-1645

58. Berti-Mattera LN, Kern TS, Siegel RE, Nemet I, Mitchell R (2008) Sulfasalazine blocks the development of tactile allodynia in diabetic rats. Diabetes 57:2801-2808

59. Østergaard L, Terkelsen AJ, Finnerup NB et al (2014) Capillary dysfunction and impaired tissue oxygenation in complex regional pain syndrome: a hypothesis. Pain 155:1922-1926

60. Watkins PJ, Edmonds ME (1983) Sympathetic nerve failure in diabetes. Diabetologia 25:73-77

61. Gabbay IE, Gabbay M, Gabbay U (2014) Diabetic foot cellular hypoxia may be due to capillary shunting-a novel hypothesis. Med Hypotheses 82:57-59

62. Archer AG, Roberts VC, Watkins PJ (1984) Blood flow patterns in painful diabetic neuropathy. Diabetologia 27:563-567

63. Kobayashi T, Puro DG (2007) Loss of insulin-mediated vasoprotection: early effect of diabetes on pericyte-containing microvessels of the retina. Invest Ophthalmol Vis Sci 48:2350-2355

64. Geraldes P, Hiraoka-Yamamoto J, Matsumoto M et al (2009) Activation of PKC-delta and SHP-1 by hyperglycemia causes vascular cell apoptosis and diabetic retinopathy. Nat Med 15:12981306

65. Peppiatt CM, Howarth C, Mobbs P, Attwell D (2006) Bidirectional control of CNS capillary diameter by pericytes. Nature 443:700 704

66. Hall CN, Reynell C, Gesslein B et al (2014) Capillary pericytes regulate cerebral blood flow in health and disease. Nature 508:5560

67. Kawamura H, Sugiyama T, Wu DM et al (2003) ATP: a vasoactive signal in the pericyte-containing microvasculature of the rat retina. $\mathrm{J}$ Physiol 551:787-799

68. Yamanishi S, Katsumura K, Kobayashi T, Puro DG (2006) Extracellular lactate as a dynamic vasoactive signal in the rat retinal microvasculature. Am J Physiol Heart Circ Physiol 290:H925-H934

69. Puro DG (2007) Physiology and pathobiology of the pericytecontaining retinal microvasculature: new developments. Microcirculation 14:1-10

70. Siperstein MD, Unger RH, Madison LL (1968) Studies of muscle capillary basement membranes in normal subjects, diabetic, and prediabetic patients. J Clin Invest 47:1973-1999 
71. Reske-Nielsen E, Lundbaek K, Rafaelsen OJ (1966) Pathological changes in the central and peripheral nervous system of young longterm diabetics: I. Diabetic encephalopathy. Diabetologia 1:233-241

72. Attwell D, Buchan AM, Charpak S, Lauritzen M, Macvicar BA, Newman EA (2010) Glial and neuronal control of brain blood flow. Nature 468:232-243

73. Kawamura H, Kobayashi M, Li Q et al (2004) Effects of angiotensin II on the pericyte-containing microvasculature of the rat retina. J Physiol 561:671-683

74. Schonfelder U, Hofer A, Paul M, Funk RH (1998) In situ observation of living pericytes in rat retinal capillaries. Microvasc Res 56: 22-29

75. Diaz-Flores L, Gutierrez R, Madrid JF et al (2009) Pericytes. Morphofunction, interactions and pathology in a quiescent and activated mesenchymal cell niche. Histol Histopathol 24:909-969

76. Lundberg JO, Weitzberg E, Gladwin MT (2008) The nitratenitrite-nitric oxide pathway in physiology and therapeutics. Nat Rev Drug Discov 7:156-167

77. Carter P, Gray LJ, Troughton J, Khunti K, Davies MJ (2010) Fruit and vegetable intake and incidence of type 2 diabetes mellitus: systematic review and meta-analysis. BMJ 341:c4229

78. Yuen KC, Baker NR, Rayman G (2002) Treatment of chronic painful diabetic neuropathy with isosorbide dinitrate spray: a double-blind placebo-controlled cross-over study. Diabetes Care 25:1699-1703

79. Malik RA, Williamson S, Abbott C et al (1998) Effect of angiotensin-converting-enzyme (ACE) inhibitor trandolapril on human diabetic neuropathy: randomised double-blind controlled trial. Lancet 352:1978-1981

80. Coppey LJ, Davidson EP, Rinehart TW et al (2006) ACE inhibitor or angiotensin II receptor antagonist attenuates diabetic neuropathy in streptozotocin-induced diabetic rats. Diabetes 55:341-348

81. Manschot SM, Gispen WH, Kappelle LJ, Biessels GJ (2003) Nerve conduction velocity and evoked potential latencies in streptozotocin-diabetic rats: effects of treatment with an angiotensin converting enzyme inhibitor. Diabetes Metab Res Rev 19: 469-477

82. Kappelle AC, Biessels GJ, van Buren T, Erkelens DW, de Wildt DJ, Gispen WH (1993) Effects of nimodipine on sciatic nerve blood flow and vasa nervorum responsiveness in the diabetic rat. Eur J Pharmacol 250:43-49

83. Kappelle AC, Biessels G, Bravenboer B et al (1994) Beneficial effect of the $\mathrm{Ca}^{2+}$ antagonist, nimodipine, on existing diabetic neuropathy in the BB/Wor rat. Br J Pharmacol 111:887-893

84. Martin CL, Albers JW, Pop-Busui R, DCCT/EDIC Research Group (2014) Neuropathy and related findings in the diabetes control and complications trial/epidemiology of diabetes interventions and complications study. Diabetes Care 37:31-38

85. Hernandez N, Torres SH, Finol HJ, Vera O (1999) Capillary changes in skeletal muscle of patients with essential hypertension. Anat Rec 256:425-432

86. Corvera S, Gealekman O (2014) Adipose tissue angiogenesis: impact on obesity and type-2 diabetes. Biochim Biophys Acta 1842: 463-472

87. Asghar O, Petropoulos IN, Alam U et al (2014) Corneal confocal microscopy detects neuropathy in subjects with impaired glucose tolerance. Diabetes Care 37:2643-2646
88. Ziegler D, Papanas N, Zhivov A et al (2014) Early detection of nerve fiber loss by corneal confocal microscopy and skin biopsy in recently diagnosed type 2 diabetes. Diabetes 63:2454-2463

89. Capone C, Faraco G, Park L, Cao X, Davisson RL, Iadecola C (2011) The cerebrovascular dysfunction induced by slow pressor doses of angiotensin II precedes the development of hypertension. Am J Physiol Heart Circ Physiol 300:H397-H407

90. Mazzoni MC, Schmid-Schonbein GW (1996) Mechanisms and consequences of cell activation in the microcirculation. Cardiovasc Res 32:709-719

91. Barnes AJ, Locke P, Scudder PR, Dormandy TL, Dormandy JA, Slack J (1977) Is hyperviscosity a treatable component of diabetic microcirculatory disease? Lancet 2:789-791

92. Wiggin TD, Sullivan KA, Pop-Busui R, Amato A, Sima AA, Feldman EL (2009) Elevated triglycerides correlate with progression of diabetic neuropathy. Diabetes 58:1634-1640

93. Katsiki N, Athyros VG, Karagiannis A, Mikhailidis DP (2013) The role of statins in the treatment of type 2 diabetes mellitus: an update. Curr Pharm Des 20:3665-3674

94. Davis TM, Yeap BB, Davis WA, Bruce DG (2008) Lipid-lowering therapy and peripheral sensory neuropathy in type 2 diabetes: the Fremantle Diabetes Study. Diabetologia 51:562-566

95. Nielsen SF, Nordestgaard BG (2014) Statin use before diabetes diagnosis and risk of microvascular disease: a nationwide nested matched study. Lancet Diabetes Endocrinol 2:894-900

96. Ii M, Nishimura H, Kusano KF et al (2005) Neuronal nitric oxide synthase mediates statin-induced restoration of vasa nervorum and reversal of diabetic neuropathy. Circulation 112:93-102

97. Malik RA, Tesfaye S, Ziegler D (2013) Medical strategies to reduce amputation in patients with type 2 diabetes. Diabet Med 30:893-900

98. Albaugh G, Bellavance E, Strande L, Heinburger S, Hewitt CW, Alexander JB (2004) Nicotine induces mononuclear leukocyte adhesion and expression of adhesion molecules, VCAM and ICAM, in endothelial cells in vitro. Ann Vasc Surg 18:302-307

99. Yong T, Zheng MQ, Linthicum DS (1997) Nicotine induces leukocyte rolling and adhesion in the cerebral microcirculation of the mouse. J Neuroimmunol 80:158-164

100. Tesfaye S, Chaturvedi N, Eaton SE et al (2005) Vascular risk factors and diabetic neuropathy. N Engl J Med 352:341-350

101. Nappo F, de Rosa N, Marfella R et al (1999) Impairment of endothelial functions by acute hyperhomocysteinemia and reversal by antioxidant vitamins. JAMA 281:2113-2118

102. Ambrosch A, Dierkes J, Lobmann R et al (2001) Relation between homocysteinaemia and diabetic neuropathy in patients with type 2 diabetes mellitus. Diabet Med 18:185-192

103. Wile DJ, Toth C (2010) Association of metformin, elevated homocysteine, and methylmalonic acid levels and clinically worsened diabetic peripheral neuropathy. Diabetes Care 33:156-161

104. Lee J, Wu W, Jiang JY, Zhu B, Boas DA (2012) Dynamic light scattering optical coherence tomography. Opt Express 20:2226222277

105. Sakadzic S, Roussakis E, Yaseen MA et al (2010) Two-photon highresolution measurement of partial pressure of oxygen in cerebral vasculature and tissue. Nat Methods 7:755-759

106. Mouridsen K, Hansen MB, Østergaard L, Jespersen SN (2014) Reliable estimation of capillary transit time distributions using DSC-MRI. J Cereb Blood Flow Metab 34:1511-1521 